- Hederol $\begin{aligned} & \text { Health and demographic research } \\ & \text { Ghent University }\end{aligned}$

sis.

$(2,1)$

LUND

UNIVERSITY

EDUCATIONAL HETEROGAMY AND THE DIVISION OF PAID LABOUR IN THE FAMILY: A COMPARISON OF PRESENT-DAY BELGIUM AND SWEDEN

Mieke Eeckhaut ${ }^{1}$,

Maria Stanfors'2,

Bart Van de Putte ${ }^{1}$

European Population Conference, Stockholm June 14, 2012

${ }^{1}$ Department of Sociology, Ghent University, Belgium

${ }^{2}$ Center for Economic Demography, Lund University, Sweden

Correspondence: Mieke Eeckhaut, Korte Meer 5, 9000 Ghent (Belgium) - Mieke.Eeckhaut@UGent.be

\title{
STRATIFICATION RESEARCH
}

Family as unit of analysis

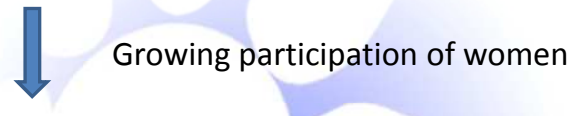

Individual as unit of analysis

Individual \& partner

Growing interest in the partner

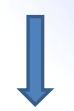

'Real' couple perspective

Couple effects?!

LUND

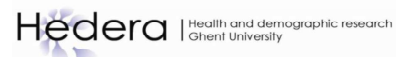




\section{RELATIVE FEMALE LABOUR MARKET PARTICIPATION}

1. What is the role of the educational composition of the couple for relative female labour market participation?

$>$ Specialization hypothesis (cf. specialization theory, bargaining theory)

$>$ Gender Identity hypothesis (cf. gender identity theory)

2. What is the role of the presence of (young) children for relative female labour market participation?

$>$ Direct Child Effect hypothesis

$>$ Indirect Child Effect hypothesis

BELGIUM VS. SWEDEN

Belgium

Conservative welfare state

$\rightarrow$ Male breadwinner model

Educational composition?

$>$ Specialization hypothesis

$\mathrm{BE}<\mathrm{SE}$

$>$ Gender Identity hypothesis

$\mathrm{BE}>\mathrm{SE}$

The presence of (young) children?

$>$ Direct Child Effect hypothesis

$\mathrm{BE}<\mathrm{SE}$

$>$ Indirect Child Effect hypothesis

$\mathrm{BE}<\mathrm{SE}$

\section{Sweden}

$\rightarrow$ Dual-earner family 
DATA

- EU-SILC 2004-2008

- Pooled cross-sectional data

- 2,592 Belgian and 3,348 Swedish couples:

- Never-married (cohabiting) or married

- Both partners born in BE/SE

- Between 25-65 years old

- Two adult household

- Not: disabled, student, unpaid work

- Variables:
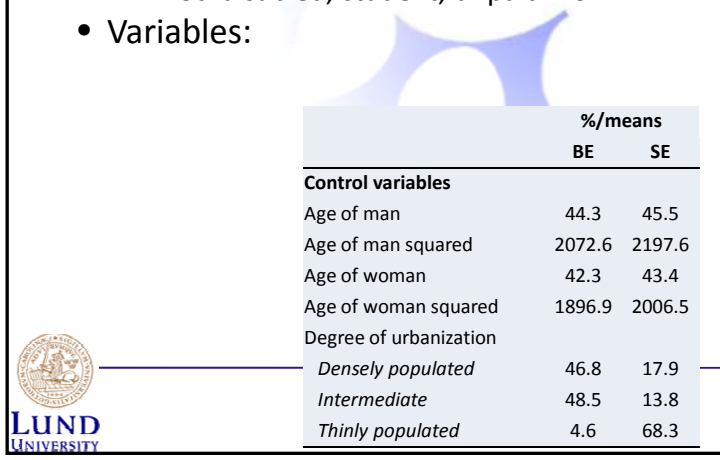

\begin{tabular}{|c|c|c|}
\hline & \multicolumn{2}{|c|}{$\% /$ means } \\
\hline & BE & SE \\
\hline \multicolumn{3}{|l|}{ Independent variables } \\
\hline \multicolumn{3}{|l|}{ Man's education } \\
\hline Primary & 7.0 & 5.6 \\
\hline Lower secondary & 14.7 & 9.3 \\
\hline (Upper) secondary & 36.5 & 47.7 \\
\hline Post-secondary, non-tertiary & 3.4 & 8.3 \\
\hline Tertiary & 38.4 & 29.1 \\
\hline \multicolumn{3}{|l|}{ Woman's education } \\
\hline Primary & 8.5 & 2.6 \\
\hline Lower secondary & 12.9 & 5.7 \\
\hline (Upper) secondary & 32.7 & 47.0 \\
\hline Post-secondary, non-tertiary & 2.4 & 4.8 \\
\hline Tertiary & 43.4 & 40.0 \\
\hline Educational heterogamy & -0.1 & -0.3 \\
\hline \multicolumn{3}{|l|}{ Presence of (young) children } \\
\hline No dependent children & 42.3 & 43.8 \\
\hline Youngest child $6-18$ years & 32.1 & 29.8 \\
\hline Youngest child $<6$ years & 25.2 & 26.3 \\
\hline \multicolumn{3}{|l|}{ Dependent variables } \\
\hline \multicolumn{3}{|c|}{ Woman's share of couple working hours } \\
\hline $0 \%$ & 18.3 & 8.6 \\
\hline $1-40 \%$ & 24.4 & 16.0 \\
\hline $41-59 \%$ & 37.5 & 63.8 \\
\hline$\geq 60 \%$ & 6.9 & 8.0 \\
\hline
\end{tabular}

Diagonal Reference Models

$$
\begin{aligned}
& Y_{\mathrm{ijk}}=p^{*} \mu_{\mathrm{ij}}+(1-p) * \mu_{\mathrm{ij}}+\sum B_{1} * H_{\mathrm{ijl}}+\sum B_{\mathrm{c}} * X_{\mathrm{ijc}}+\sum B_{\mathrm{h}} * H_{\mathrm{ijh}}+\varepsilon_{\mathrm{ijk}} \quad \mathrm{i}=1, \ldots, \mathrm{T} ; \\
& \underbrace{\mathrm{j}=1, \ldots, \mathrm{T} \text {; }} \\
& \begin{array}{ccc}
\text { Control } & \text { Presence of Educational } & \mathrm{k}=1, \ldots, n_{\mathrm{ij}} \\
\text { variables (young) children heterogamy } &
\end{array}
\end{aligned}
$$

Education woman

\begin{tabular}{|c|c|c|c|c|c|}
\cline { 2 - 6 } Education man & $\mathbf{1}$ & $\mathbf{2}$ & $\mathbf{3}$ & $\mathbf{4}$ & $\mathbf{5}$ \\
\hline $\mathbf{1}$ & $\boldsymbol{\mu}_{11}$ & & & & \\
\hline $\mathbf{2}$ & & $\boldsymbol{\mu}_{22}$ & & & \\
\hline 3 & & & $\boldsymbol{\mu}_{33}$ & & \\
\hline 4 & & & & $\boldsymbol{\mu}_{44}$ & \\
\hline $\mathbf{5}$ & & & & & $\boldsymbol{\mu}_{55}$ \\
\hline
\end{tabular}

\section{$\rightarrow$ Multinomial logistic DRMs}




\section{RESULTS -}

Multinomial logistic Diagonal Reference Models

1. Baseline Model + Control Variables

2. + Presence of (Young) Children + Educational Heterogamy

$Y_{\mathrm{ijk}}=p^{*} \mu_{\mathrm{ii}}+(1-p) * \mu_{\mathrm{jj}}+\sum B_{1} * X_{\mathrm{ijl}}+\sum B_{\mathrm{c}} * X_{\mathrm{ijc}}+\sum B_{\mathrm{h}} * H_{\mathrm{ijh}}+\varepsilon_{\mathrm{ijk}}$

Results 1 - BASELINe Model + CV

\begin{tabular}{|c|c|c|c|c|c|c|c|c|}
\hline & \multicolumn{4}{|c|}{ Belgium } & \multicolumn{4}{|c|}{ Sweden } \\
\hline & & \multicolumn{6}{|c|}{ Salience Parameter $p$} & \\
\hline & & \multicolumn{3}{|c|}{0.033} & \multicolumn{3}{|c|}{0.216} & \\
\hline & \multicolumn{8}{|c|}{ Odds for the Homogamous with Educational Level i (Probability Between Brackets) } \\
\hline & $0 \%$ & $1-40 \%$ & $41-59 \%$ & $>59 \%$ & $0 \%$ & $1-40 \%$ & $41-59 \%$ & $>59 \%$ \\
\hline \multirow[t]{2}{*}{$\mu_{11}$} & 1.269 & $0.454^{*}$ & 1 & 0.742 & $2.661^{*}$ & 0.550 & 1 & $0.130 *$ \\
\hline & $(36.6 \%)$ & (13.1\%) & $(28.9 \%)$ & $(21.4 \%)$ & $(61.3 \%)$ & $(12.7 \%)$ & $(23.0 \%)$ & $(3.0 \%)$ \\
\hline \multirow[t]{2}{*}{$\mu_{22}$} & 0.576 & $0.212 *$ & 1 & $0.221 *$ & 0.557 & $0.223 *$ & 1 & $0.138 *$ \\
\hline & $(28.7 \%)$ & $(10.6 \%)$ & $(49.8 \%)$ & $(11.0 \%)$ & $(29.0 \%)$ & (11.6\%) & $(52.2 \%)$ & $(7.2 \%)$ \\
\hline \multirow[t]{2}{*}{$\mu_{33}$} & $0.309 *$ & 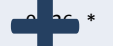 & & $0.198 *$ & $0.515 *$ & " כול ع * & 1 & $0.097 *$ \\
\hline & $(17.8 \%)$ & $(13 . \omega)$ & $(57.7 \%)$ & $(11.4 \%)$ & $(28.7 \%)$ & $(10.2 \%)$ & $(55.7 \%)$ & $(5.4 \%)$ \\
\hline \multirow[t]{2}{*}{$\mu_{44}$} & 0.701 & $0.272 *$ & 1 & 0.737 & 0.460 & $0.122 *$ & 1 & $0.066 *$ \\
\hline & $(25.9 \%)$ & $(10.0 \%)$ & $(36.9 \%)$ & $(27.2 \%)$ & $(27.9 \%)$ & $(7.4 \%)$ & $(60.7 \%)$ & $(4.0 \%)$ \\
\hline \multirow[t]{2}{*}{$\mu_{55}$} & $0.142 *$ & $0.213 *$ & 1 & $0.162 *$ & $0.273 *$ & $0.118 *$ & 1 & $0.097 *$ \\
\hline & $(9.3 \%)$ & (14.1\%) & $(65.9 \%)$ & $(10.7 \%)$ & $(18.4 \%)$ & $(7.9 \%)$ & $(67.2 \%)$ & $(6.5 \%)$ \\
\hline$b_{\text {age woman }}$ & $0.896^{*}$ & 1.032 & 1 & 0.981 & $0.790 *$ & 0.999 & 1 & 0.936 \\
\hline$b_{\text {age woman squared }}$ & $1.004 *$ & 0.999 & 1 & 0.999 & $1.006 *$ & 1.000 & 1 & 1.000 \\
\hline$b_{\text {age man }}$ & 1.057 & 1.076 & 1 & $0.866 *$ & 1.057 & 1.021 & 1 & 0.895 \\
\hline$b_{\text {age man squared }}$ & 1.000 & 0.999 & 1 & $1.007 *$ & 0.998 & 0.999 & 1 & $1.005 *$ \\
\hline$b_{\text {intermediate populated }}$ & 1.219 & 1.144 & 1 & 1.001 & 0.859 & 1.142 & 1 & 1.325 \\
\hline$b_{\text {thinly populated }}$ & 1.315 & $1.645 *$ & 1 & 0.729 & 0.926 & $1.469 *$ & 1 & 1.396 \\
\hline$N$ & \multicolumn{4}{|c|}{2,254} & \multicolumn{4}{|c|}{3,224} \\
\hline
\end{tabular}


Results 2 - BASELINE MOdEL + CV + PRESENCE OF (YOUng) ChILdREN + Educational Heterogamy

\begin{tabular}{|c|c|c|c|c|c|c|c|c|}
\hline & \multicolumn{8}{|c|}{ Odds for the Presence of (Young) Children and Educational Heterogamy } \\
\hline & \multicolumn{4}{|c|}{ Belgium } & \multicolumn{4}{|c|}{ Sweden } \\
\hline & $0 \%$ & $1-40 \%$ & $41-59 \%$ & $>59 \%$ & $0 \%$ & $1-40 \%$ & $41-59 \%$ & $>59 \%$ \\
\hline$b_{\text {youngest child } 6-18 y}$ & $2.481 *$ & $1.740^{*}$ & 1 & 1.064 & $2.143 *$ & $1.653^{*}$ & 1 & $1.566 *$ \\
\hline$b_{\text {youngest child < } 6 y}$ & $3.858 *$ & $2.540 *$ & 1 & 1.660 & $9.929 *$ & $1.868 *$ & 1 & $2.179^{*}$ \\
\hline$b_{\text {educ. heterogamy }}$ & 1.014 & 0.993 & 1 & 0.888 & $1.392 *$ & 1.184 & 1 & 1.000 \\
\hline
\end{tabular}

Educational Heterogamy

BE: No significant effects

SE: Specialization hypothesis, BUT limited to $0 \%$ vs. $41-59 \%$

Presence of (Young) Children

BE: (Young) children = higher odds of working $0 \%$ and $1-40 \%$

SE: (Young) children = higher odds of working $0 \%, 1-40 \%$, and $>59 \%$

Interaction

BE: No interaction effect

SE: Woman without dependent children $\gg$ Woman with children $<6 y$

\section{CONCLUSION}

Female labour market participation

BE

$<<$

Education woman

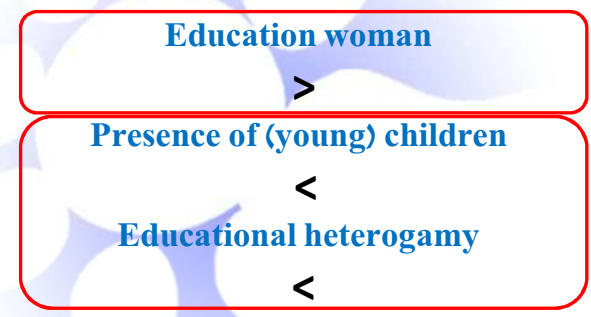

More egalitarianism and family-friendliness in SE

Stronger effect of age and education in BE

Small effect of educational heterogamy overall (!) 


\section{THANK YOU FOR YOUR ATTENTION!}

Correspondence: Mieke Eeckhaut, Korte Meer 5, 9000 Ghent (Belgium) - Mieke.Eeckhaut@UGent.be
LUNIVERSIIYY 